\title{
Estresse ocupacional e burnout em profissionais de saúde de unidades de perioperatório
}

Occupational stress and burnout in health professionals of perioperative units Estrés laboral y burnout en profesionales de la salud de unidades de perioperatorio

\author{
Oclaris Lopes Munhoz ${ }^{1}$ it hitps://orcidi.org/0000-0001-8901-7148 \\ Tatiele Soares Arrial ${ }^{1}$ if https://orcid.org/0000-0002-5184-4639 \\ Edison Luiz Devos Barlem² iD https://orcid.org/0000-0001-6239-8657 \\ Graziele de Lima Dalmolin ${ }^{1}$ io https://orcid.org/0000-0003-0985-5788 \\ Rafaela Andolhe ${ }^{1}$ io https://orid. org/0000-0003-3000-8188 \\ Tânia Solange Bosi de Souza Magnago ${ }^{1}$ io hittps://orcid.org/0000-0002-5308-1604
} Andolhe R, Magnago TS. Estresse ocupacional e burnout em profissionais de saúde de unidades de perioperatório. Acta Paul Enferm. 2020;33:AAPE20190261.

DOI

http://dx.doi.org/10.37689/actaape/2020A00261

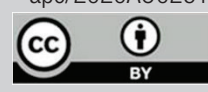

Descritores

Esgotamento psicológico; Saúde do trabalhador; Pessoal da saúde; Estresse ocupacional; Esgotamento profissional; Assistência perioperatória

Keywords

Burnout, psychological; Occupational health Health personnel; Occupational stress; Burnout, professional; Perioperative care

Descriptores

Agotamiento psicológico: Salud laboral; Personal de salud; Estrés laboral; Agotamiento profesional; Atención perioperativa

Submetido 9 de Setembro de 2019

Aceito

17 de Dezembro de 2019

Autor correspondente Oclaris Lopes Munhoz

E-mail: oclaris_munhoz@hotmail.com

\section{Resumo}

Objetivo: Analisar a relação entre estresse ocupacional e burnout em profissionais de saúde de unidades de perioperatório.

Métodos: Estudo transversal analítico, desenvolvido com 146 profissionais de saúde de unidades de perioperatório, representantes de diversas categorias. Utilizou-se os instrumentos Job Stress Scale e 0 Inventário Maslach de Burnout. Para a análise dos dados empregou-se estatística descritiva e inferencial.

Resultados: Quanto ao estresse ocupacional, constatou-se que 93(64,5\%) profissionais de saúde encontravam-se com altas demandas psicológicas e $83(57,3 \%)$ estavam com baixo controle sobre 0 trabalho. Observou-se que $15(10,3 \%)$ profissionais apresentaram burnout. Houve associação estatisticamente significativa entre alta demanda psicológica e alto desgaste emocional $(p=0,0001)$ e, entre alta demanda psicológica e alta despersonalização $(p=0,007)$. Evidenciou-se associação estatisticamente significativa entre presença de burnoute altas demandas psicológicas $(\mathrm{p}=0,049)$.

Conclusão: Profissionais desgastados emocionalmente e que se afastam dos colegas possuem altas demandas psicológicas. À medida que as demandas psicológicas aumentam, também aumentam o desgaste emocional e a despersonalização. Por outro lado, quando a demanda psicológica é baixa, o profissional não está em burnout.

\section{Abstract}

Objective: To analyze the relationship between occupational stress and burnout in healthcare professionals working in perioperative units.

Methods: Analytical, cross-sectional study developed with 146 health professionals from various categories working in perioperative units. The Job Stress Scale and the Maslach Burnout Inventory were used. Descriptive and inferential statistics were used for data analysis.

Results: Regarding occupational stress, 93 (64.5\%) health professionals had high psychological demands and 83 (57.3\%) had low control over work. Fifteen (10.3\%) professionals were burnout. There was a statistically significant association between high psychological demand and high emotional exhaustion $(p=0.0001)$ and between high psychological demand and high depersonalization $(\mathrm{p}=0.007$ ). There was a statistically significant association between the presence of burnout and high psychological demands $(p=0.049)$.

Conclusion: Emotionally strained professionals who move away from colleagues have high psychological demands. As psychological demands increase, so does emotional exhaustion and depersonalization. On the other hand, when the psychological demand is low, the professional is not experiencing burnout. 


\section{Resumen}

Objetivo: Analizar la relación entre el estrés laboral y burnout en profesionales de la salud de unidades de perioperatorio.

Métodos: Estudio transversal analítico, realizado con 146 profesionales de la salud de unidades de perioperatorio, representantes de diferentes categorías. Se utilizaron los instrumentos Job Stress Scale y el Cuestionario Maslach de Burnout. Para analizar los datos se aplicó estadística descriptiva e inferencial.

Resultados: Respecto al estrés laboral, se constató que 93 (64,5\%) profesionales de la salud se encontraban con altas demandas psicológicas y 83 (57,3\%) tenían un bajo control sobre el trabajo. Se observó que 15 (10,3\%) profesionales presentaron burnout. Hubo relación estadísticamente significativa entre la alta demanda psicológica y el alto desgaste emocional $(\mathrm{p}=0,0001)$, y entre la alta demanda psicológica y la alta despersonalización ( $\mathrm{p}=0,007)$. Se constató relación estadísticamente significativa entre presencia de burnouty altas demandas psicológicas $(p=0,049)$.

Conclusión: Profesionales desgastados emocionalmente y que se alejan de los compañeros tienen altas demandas psicológicas. A medida que las demandas psicológicas aumentan, también aumenta el desgaste emocional y la despersonalización. Por otro lado, cuando la demanda psicológica es baja, el profesional no está en estado de burnout.

\section{Introdução}

Os ambientes de trabalho são considerados, muitas vezes, locais desgastantes. Fatores como o estresse laboral e o burnout comprometem a saúde dos profissionais. Assim, a necessidade de estudos que abordam o impacto do estresse na saúde dos trabalhadores e sua repercussão nas atividades assistenciais têm crescido nas últimas décadas, visto que além da saúde do trabalhador, a segurança do paciente também é comprometida. ${ }^{(1,2)}$

Nesta perspectiva, dentre os modelos teóricos desenvolvidos para avaliar o estresse referente ao ambiente de trabalho, de natureza psicossocial, o modelo demanda-controle (MD-C) está entre os mais utilizados. Este modelo busca elucidar as exigências mentais que implicam no trabalho do profissional, sendo assim, quanto maior as exigências do trabalho, maior é a exigência psicológica do profissional. $(3,4) \mathrm{O}$ excesso de demandas psicológicas, de grande complexidade e que necessitam serem feitas em pouco tempo, aumentam as exigências psicológicas. Tais demandas envolvem: pressão do tempo, nível de concentração, interrupção de tarefas e necessidade de depender de outros. $\mathrm{O}$ controle no trabalho é a possibilidade que o profissional tem de usar habilidades (criatividade e estratégias) e a tomada de decisão para realizar as suas demandas, combatendo os estressores. ${ }^{(3,4)}$

Por conseguinte, quando os profissionais não conseguem ter controle sobre o seu trabalho e náo utilizam estratégias de enfrentamento, podem acabar adoecendo, podendo ser acometidos pelo burnout. ${ }^{(1)} \mathrm{O}$ burnout está relacionado às consequências do trabalho na saúde dos profissionais e atinge, principalmente, os trabalhadores que atuam em contato direto com os pacientes, sendo reconhecido por ser um processo de risco ocupacional para as profissóes que envolvem educação, cuidados e serviços com humanos. ${ }^{(5,6)}$

Dentre as concepçóes teóricas que definem um modelo explicativo para o Burnout, está a Concepção Socioambiental, que evidencia fatores sócio ambientais como precursores do seu desenvolvimento. Assim, fatores oriundos do ambiente trabalho podem fragilizar um indivíduo, fazendo com que este desencadeie a síndrome. ${ }^{(5,7)} \mathrm{O}$ modelo socioambiental, elenca fatores multidimensionais do burnout e considera aspectos individuais, associados às condiçóes e relaçôes do trabalho. Tais fatores se subdividem em três dimensôes: desgaste emocional, relacionado ao esgotamento físico, mental e emocional; despersonalização, indicando que a personalidade do indivíduo está sofrendo alteraçôes como consequência do seu trabalho e; realização profissional, que evidencia a satisfação com as atividades laborais. ${ }^{(5,7)}$

A realização deste estudo justifica-se em razão de que os ambientes de perioperatório estão entre os cenários de atuação de profissionais da saúde que mais possuem estressores, resultado da complexidade dos procedimentos que são realizados e da competência e autonomia exigida neste tipo de ambiente. ${ }^{(1,8,9)}$ Neste contexto, estudo constatou que os profissionais atuantes no cenário cirúrgico não estavam satisfeitos com o trabalho, não estavam dispostos a trabalhar, estavam cansados, com poucas horas de sono e consideravam os recursos materiais insuficientes, demostrando assim, uma maior suscetibilidade para o desenvolvimento de estresse e bur- 
nout. ${ }^{(1)}$ Outra pesquisa constatou que a unidade de clínica cirúrgica foi o setor em que predominaram os maiores níveis de estresse entre os profissionais. ${ }^{(9)}$

Nesse sentido, pesquisas acerca do estresse e burnout, medidas de estresse agudo e crônico, respectivamente, têm contribuído para a identificação dos fatores associados ao adoecimento do profissional, com consequências para a segurança do paciente. ${ }^{(1,2,6)}$ Contudo, são escassos os estudos direcionados a esses fatores em unidades de perioperatório, as quais possuem peculiaridades que exigem preparação profissional para o atendimento aos pacientes cirúrgicos.

Frente a essas consideraçóes, questiona-se: existe relação entre estresse ocupacional e burnout em profissionais de saúde de unidades de perioperatório? Entende-se que quando o indivíduo apresenta baixo controle e altas demandas psicológicas em relação ao estresse ocupacional, é possível que essa situação se caracterize pela inicial cronificação do estresse e, portanto, ele esteja mais suscetível ao burnout. Assim, a hipótese deste estudo é de que profissionais com baixo controle e altas demandas psicológicas estão em burnout. Este artigo tem por objetivo analisar a relação entre estresse ocupacional e burnout em profissionais de saúde de unidades de perioperatório.

\section{Métodos}

\section{Tipo de estudo}

Tratou-se de um estudo transversal analítico, realizado com profissionais de saúde de um hospital escola da regiáo central do Rio Grande do Sul.

\section{Cenário e população}

$\mathrm{O}$ estudo foi desenvolvido nas unidades de perioperatório, que correspondem ao Bloco Cirúrgico (BC), a Sala de Recuperação Anestésica (SRA), a Sala de Recuperação Intermediária (SRI) e a Unidade de Cirurgia Geral - Serviço de Internação (UCG-SI). Essas unidades prestam cuidados aos pacientes com necessidade de intervenção cirúrgica, nos períodos pré, intra e pós-operatório. Os participantes do estudo foram: equipe de enfermagem, médicos, psicó- logos, assistentes sociais, fisioterapeutas, dentistas, fonoaudiólogos, nutricionistas e auxiliares de saúde.

\section{Critérios de seleção e definição da população}

Definiu-se como critérios de seleção da população: profissionais de saúde com no mínimo quatro semanas de atuação e carga horária mínima de 20 horas semanais na unidade de lotação. Estes critérios estão são necessários para a avaliação do burnout. ${ }^{(7)}$ Foram excluídos os profissionais afastados no período da coleta por qualquer motivo. Constitui-se de uma população por conveniência e não probabilística, na qual de um total de 181 profissionais atuantes nas unidades de perioperatório, participaram da pesquisa 146 profissionais de saúde, representando 80,7 \% da população elegível.

\section{Coleta de dados e instrumentos utilizados}

Os dados foram coletados no período de março a julho de 2018, após tramitação ética e autorização institucional. Anteriormente ao início das coletas, realizou-se um contato com as gerências das unidades estudadas, visando informar os objetivos e convidar os profissionais para o estudo. Após consentimento assinado por meio do Termo de Consentimento Livre e Esclarecido (TCLE), os profissionais de saúde foram convidados a participar da pesquisa e a preencherem os instrumentos de coleta em seu local de trabalho, em local reservado, nas suas respectivas unidades. Durantes as coletas, entregava-se um envelope contendo a Job Stress Scale (JSS) e o Maslach Burnout Inventory-Human Service Survey (MBI-HSS). ${ }^{(4,7)}$ Utilizou-se o instrumento JSS para a avaliação do estresse ocupacional, em sua versão reduzida e adaptada para a realidade brasileira com alpha de Cronbach entre 0,63 e 0,86. ${ }^{(4)}$ Esta versão possui 17 questôes, divididas em três domínios: o primeiro representa a demanda psicológica (DP), avaliada por cinco questóes, por meio de uma escala do tipo Likert, com opçôes de respostas para frequentemente, às vezes, raramente e nunca; o segundo é o controle (C), interpretado por seis questôes em escala tipo Likert (1-4), também variando de frequentemente a nunca; o terceiro domínio é o de apoio social (AS), que não foi utilizado, pois não foi objetivo de análise desta pesquisa. Para o do- 
mínio DP a pontuação pode variar de no mínimo cinco até no máximo 20 pontos (quanto maior o escore maior a demanda psicológica). Para o domínio $\mathrm{C}$, a variação pode ocorrer de seis até 24 pontos (quanto maior o escore maior o controle). As questôes de número 4 e 9 possuem pontuação reversa. ${ }^{(4)}$ Para o levantamento do nível de burnout utilizou-se o IMB-HSS, com alpha de Cronbach de 0,89. Este instrumento possuí 22 itens, subdivididos nas dimensóes desgaste emocional (DE), despersonalização (DP) e realização profissional (RP). Para todas opçóes de resposta existe uma escala do tipo Likert que varia de zero "nunca" a quatro "diariamente", na qual o profissional marca a frequência com que se percebe ou sente-se com relação ao enunciado de cada questão. ${ }^{(6)}$

\section{Análise e tratamento dos dados}

Os dados foram digitados em planilhas Excel e processados no programa Statistical Package for the Social Sciences (SPSS), versão 18.0, com validação e checagem da consistência. Para a identificação da associação entre estresse ocupacional e burnout utilizou-se o teste Exato de Fischer ou Qui-Quadrado. Para as correlaçóes entre as variáveis quantitativas utilizou-se a correlação de Pearson. A normalidade dos dados foi analisada pelo teste Kolmogorov-Smirnov. Considerou-se associaçóes estatisticamente significativas quando o valor de $\mathrm{p}$ foi menor ou igual a 0,05 . Conforme a distribuição dos dados, utilizou-se a mediana para a dicotomizaçáo dos domínios da JSS. Os valores obtidos foram categorizados a partir de cada dimensão, correspondendo a baixo e alta demanda psicológica $(7-13$ pontos = Baixa DP; $14-19$ pontos = Alta DP) e baixo e alto controle $(11-18$ pontos = Baixo C; $19-22$ pontos = Alto C). Para a análise do burnout, os pontos de corte para os domínios foram obtidos por tercis, conforme o recomendado pelo Manual do MBI. ${ }^{(7)} \mathrm{O}$ tercil estabelece o ponto de corte da amostra, em cada subescala. Pontos de corte da amostra estudada: Desgaste Emocional: Baixo DE $\leq$ 6; Médio $\mathrm{DE}=$ entre 6,1 e 10,9; Alto $\mathrm{DE} \geq 11$; Despersonalização: Baixa $\mathrm{DP} \leq 1$; Média $\mathrm{DP}=$ entre 1,1 e 2,9; Alta DP $\geq 3$; Realizaçáo Profissional: Baixa $\mathrm{RP} \leq 20$; Média RP = entre 20,1 e 23,9; Alta RP $\leq$ 24. O intervalo estabelecido pelos tercis é importante para determinar os critérios que indicam a presença de burnout, ou seja: quando há altas pontuações de desgaste emocional e despersonalização, associadas à baixa pontuação de realização profissional, a pessoa está em burnout. ${ }^{(7)}$

\section{Aspectos éticos}

Este estudo coaduna-se com a Resolução 466/2012 do Conselho Nacional de Saúde, visto que foi aprovado pelo Comitê de Ética em Pesquisa da Universidade Federal de Santa Maria, em dezembro de 2017, sob o parecer no 2.447.277 e CAAE: 80587417.0.0000.5346.

\section{Resultados}

Dos profissionais de saúde participantes deste estudo, 93(64,5\%) encontravam-se com altas demandas psicológicas e outros 51(35,5\%) com baixas demandas psicológicas. Ademais, 83(57,3\%) estavam com baixo controle sobre o trabalho e $62(42,7 \%)$ com alto controle sobre o trabalho. Ao analisar a presença de burnout entre os profissionais de saúde das unidades de perioperatório, observou-se que 15(10,3\%) apresentaram a síndrome. A tabela 1 apresenta as associaçóes entre demanda psicológica $\mathrm{e}$ as dimensões de burnout.

Tabela 1. Associação entre demanda psicológica e as dimensões de burnout

\begin{tabular}{lcccc}
\hline \multirow{2}{*}{ Demanda psicológica } & \multicolumn{4}{c}{ Desgaste emocional } \\
& $\mathrm{n}(\%)$ & Médio & Alto & p-value \\
& $27(54,0)$ & $16,0(32,0)$ & $7(14,0)$ & \multirow{2}{*}{$0,0001 \dagger$} \\
\hline Baixa demanda & $20(21,5)$ & $29(31,2)$ & $44(47,3)$ & \\
Alta demanda & \multicolumn{5}{c}{ Despersonalização } \\
\hline \multirow{3}{*}{ Demanda psicológica } & Baixa & Média & Alta & p-value \\
& $\mathrm{n}(\%)$ & $\mathrm{n}(\%)$ & $\mathrm{n}(\%)$ & \\
\hline Baixa demanda & $21(41,1)$ & $16(31,4)$ & $14(27,5)$ & \multirow{2}{*}{$0,007 \dagger$} \\
Alta demanda & $25(26,9)$ & $17(18,3)$ & $51(54,8)$ & \\
\hline \multirow{3}{*}{ Demanda psicológica } & Realização Profissional & Alta & -value \\
& Baixa & Média & $\mathrm{n}(\%)$ & \\
\hline Baixa demanda & $\mathrm{n}(\%)$ & $\mathrm{n}(\%)$ & $24(47,1)$ & \multirow{2}{*}{$0,540 \dagger$} \\
Alta demanda & $13(25,5)$ & $14(27,5)$ & $35(37,6)$ & \\
\hline
\end{tabular}

† Teste Qui-quadrado de Pearson

Conforme tabela 1, houve associação estatisticamente significativa entre alta demanda psicológica e alto desgaste emocional $(p=0,00001)$ e, entre 
alta demanda psicológica e alta despersonalização $(\mathrm{p}=0,007)$. Não houve associação estatisticamente significativa entre demanda psicológica e realização profissional. Com relação às análises entre controle sobre o trabalho e burnout, náo houve associação estatisticamente significativa para nenhuma dimensão $(p \geq 0,05)$. A tabela 2 demonstra a associação entre estresse ocupacional e burnout, evidenciando-se que os profissionais de saúde que estão em burnout tem altas demandas psicológicas ( $\mathrm{p}=0,049)$.

Tabela 2. Associação entre estresse ocupacional (JSS) e burnout (MBI)

\begin{tabular}{llccc}
\hline \multirow{2}{*}{ Dimensões MD-C } & & \multicolumn{3}{c}{ Burnout } \\
& & Presente & Ausente & p-value \\
\hline \multirow{2}{*}{ Controle sobre 0 Trabalho } & Baixo & $7(8,4)$ & $76(91,6)$ & \multirow{2}{*}{$0,273 \dagger$} \\
& Alto & $8(12,9)$ & $54(12,9)$ & \\
Demanda Psicológica & Baixa & $2(3,9)$ & $49(96,1)$ & \multirow{2}{*}{$0,049 ¥$} \\
& Alta & $13(14,0)$ & $80(86,0)$ & \\
\hline
\end{tabular}

†Teste Qui-quadrado de Pearson; ¥Teste exato de Fischer

\section{Discussão}

Os profissionais de saúde das unidades de perioperatório encontravam-se com altas demandas psicológicas e baixo controle sobre o trabalho. Esse panorama caracteriza-se por representar um trabalho de alta exigência, o que resulta em reaçóes adversas à saúde do profissional. Nessa situação, pode haver fadiga mental e ansiedade, fatores estes que colocam em risco a saúde do profissional como decorrência do seu ambiente de trabalho. ${ }^{(3,4)}$ Ainda, profissionais de saúde com baixo controle sobre as atividades laborais, podem não enfrentar os estressores laborais, o que resulta em risco de adoecimento. ${ }^{(1)}$

Esses achados divergem do encontrado em outro estudo, no qual prevaleceram os profissionais com baixa demanda e alto controle sobre o trabalho. Por outro lado, esse mesmo estudo constatou a unidade cirúrgica como sendo a segunda com maior prevalência de profissionais estressados, quando comparada à unidade de tratamento intensivo e à unidade de pronto atendimento. ${ }^{(10)}$ Entende-se que existem fatores podem estar relacionados com o estresse vivenciado nos ambientes de perioperatório, como por exemplo o alto número de procedimentos complexos que são realizados, as cirurgias de emergência e a rotatividade de pacientes. Essas situaçóes caracterizam-se como estressores e exigem dos profissionais de saúde preparo técnico e psicológico. ${ }^{(10-12)}$

Em outra investigação, os profissionais demonstraram ter altas demandas psicológicas, contudo, tinham alto controle sobre o trabalho. Isso equivale ao trabalho ativo, em que os profissionais possuem autoridade de decisão e que apesar das altas demandas, o alto controle traz benefícios à saúde deles. ${ }^{(11)}$ Nesse caso, as demandas psicológicas do ambiente de trabalho são enfrentadas como desafios, assim como proporcionam crescimento e aprendizado profissional. ${ }^{(3)}$

Ainda no que diz respeito às demandas psicológicas e ao controle sobre o trabalho evidenciado nos profissionais de saúde do estudo, é sabido que as unidades em que esses atuam envolvem altas demandas psicológicas, visto que há uma grande rotatividade de pacientes, das mais diversas complexidades, bem como o número de procedimentos realizados é alto. Essa realidade vai ao encontro do que propóem o referencial do constructo avaliado, dado que o excesso de demandas de trabalho e de alta complexidade aumentam as exigências psicológicas por parte dos profissionais. Por conseguinte, autores destacam que o controle está diretamente relacionado com a possibilidade que o profissional tem de usar habilidades (criatividade e estratégias) para dar conta de suas demandas e combater os estressores. Contudo, percebe-se que os profissionais em um percentual considerável foram classificados em baixo controle sobre as suas demandas de trabalho. ${ }^{(3,4)}$

Além disso, nesta pesquisa, 15(10,3\%) profissionais de saúde apresentaram a síndrome de burnout, achado que aproxima-se aos de outras pesquisas nas quais a prevalência de burnout foi de 12,5\% e $14,4 \% .^{(1,13)}$ Verifica-se que, se os profissionais não mantiverem o controle do estresse ocupacional, talvez a prevalência de burnout aumente, sendo relevante que a instituição mantenha estratégias de valorização profissional, bem como oportunize espaços de promoçáo ao enfrentamento do estresse.

O burnout pode desencadear consequências individuais de trabalho, de organização de trabalho e sociais. (14) $\mathrm{Na}$ esfera individual, há o desenvolvimento de dores, alergias, distúrbios gastrointestinais, falta de concentração, agressividade e baixa autoestima. No que se refere ao trabalho, o burnout aumenta as chances de erros 
assistenciais e compromete o relacionamento entre os membros da equipe. No que tange a organização do trabalho, gera aumento dos gastos em tempo e dinheiro, resultado da rotatividade dos profissionais adoecidos. Por fim, a esfera social é fragilizada, dado que o burnout leva à desarmonia familiar e torna o profissional menos satisfeito em sua relaçáo com paciente. ${ }^{(14)}$

Nesse ínterim, entende-se que a rotina das unidades estudadas, devido ao elevado número de procedimentos realizados, rotatividade de pacientes e complexidade das açóes desenvolvidas, pode ter contribuído para o desencadeamento das consequências supracitadas nos profissionais acometidos pelo burnout. Considerando que o burnout é uma forma de estresse crônico e que, muitas vezes, o profissional não percebe que está acometido por essa síndrome, existem fatores desfavoráveis e que predispóem para o desenvolvimento no burnout e outros protetivos que auxiliam no enfrentamento e prevenção. . $^{(1,15-17)}$

Os fatores desfavoráveis são, principalmente, falta de reconhecimento profissional, relaçôes interpessoais conflituosas e com a gerência direta, duplo vínculo empregatício e carga horária de trabalho elevada. ${ }^{(15,16)}$ Os fatores de proteção estão relacionados a um ambiente de trabalho organizado e com apoio das instituiçôes, ao diálogo aberto e ao apoio da chefia direta. Ademais, profissionais casados, com filhos e mais jovens estão menos propensos a desenvolver a síndrome. ${ }^{(1,16-18)}$

Constatou-se, que houve associação estatisticamente significativa entre os profissionais de saúde com alta demanda psicológica e alto desgaste emocional e, os com alta demanda psicológica e alta despersonalização. Esses achados convergem com o modelo proposto por Karasek e Theörel e por Maslach e Jackson, uma vez que um profissional desgastado emocionalmente e que se afasta dos colegas terá altas demandas psicológicas de trabalho, pois, caracterizase por uma pessoa sem energia, sem motivação para trabalhar e que evita o relacionamento interpessoal, principalmente com os colegas de trabalho. ${ }^{(3,7)}$ Somase a isso, esta análise é importante por que pode ser indicador de que pessoas com altas demandas psicológicas estão mais suscetíveis a desenvolver o burnout.

Depreende-se que o desgaste emocional e a despersonalização podem estar envolvidos com a autonomia do profissional e o seu controle sobre o tra- balho, o que na população estudada caracterizou majoritariamente profissionais com baixo controle. Por outro lado, caso esses profissionais tenham controle sobre o seu ambiente de trabalho, enfrentarão os estressores laborais com mais autonomia, o que corrobora para que não tenham exaustão emocional. ${ }^{(19)}$

Somado a isso, algumas investigaçóes evidenciaram fatores que estão associados ao alto desgaste emocional, a alta despersonalização e a altas demandas psicológicas. ${ }^{(20,21)}$ Exemplo disso, contatou-se que o excesso de demandas é um fator negativo que predispóem o desenvolvimento da síndrome de burnout, situaçáo que vai ao encontro da realidade dos profissionais de saúde deste estudo, visto que a maioria apresentou-se com altas demandas psicológicas. ${ }^{(20)}$ Ainda, a atuação em unidades assistenciais com pacientes críticos, o que também é vivenciado pelos profissionais desta pesquisa, reflete em um trabalho de alta exigência e desfavorece qualidade de vida do trabalhador. ${ }^{(2)}$ Outrossim, o burnout predispóem a ocorrência de problemas respiratórios, gastrointestinais e cardiovasculares. ${ }^{(21)}$

Também evidenciou-se que os profissionais de saúde que estão em burnout tem altas demandas psicológicas. Esse achado coaduna-se ao proposto pelos autores desses constructos, uma vez que o burnout está relacionado às consequências que o trabalho traz para os profissionais, nesse caso, as altas demandas psicológicas. ${ }^{(3,7)}$ Assim, fatores oriundos do ambiente de trabalho fragilizam o indivíduo, predispondo-o a síndrome de burnout. ${ }^{(7)}$ Quanto ao estresse ocupacional, altas demandas de trabalho favorecem o desenvolvimento do estresse ocupacional. ${ }^{(3)}$ Essas realidades foram evidenciadas na população estudada. Nesse sentido, a vulnerabilidade para o desenvolvimento do burnout é potencializada pelos estressores laborais que provocam altas demandas psicológicas no trabalhador. ${ }^{(2,19,20)}$

Posto isto, este estudo contribui para o avanço do conhecimento na área da saúde do trabalhador, em especial no que se refere aos profissionais que atuam em unidades de perioperatório, ao passo que evidencia associaçóes importantes entre estresse ocupacional, Modelo Demanda-Controle e burnout. As altas demandas psicológicas se associaram a presença de burnout entre esses trabalhadores. Por essa razão, fazem-se necessárias pesquisas envolvendo este cenário, com vistas a identificar possíveis estratégias que fa- 
voreçam o enfrentamento dos estressores e o controle dos profissionais sobre as demandas psicológicas, bem como melhorem as condições de atuação profissional. Assim, saúde do trabalhador será favorecida, bem como os cuidados prestados serão mais seguros.

\section{Conclusão}

Pode-se perceber que os ambientes de perioperatório caracterizam-se por ser estressantes e por originar um trabalho em que os profissionais têm altas demandas psicológicas, o que pode resultar em profissionais desgastados emocionalmente, que se afastam dos colegas, ou que estão em burnout, possuindo altas demandas psicológicas. Em suma, a hipótese deste estudo foi confirmada, pois profissionais com altas demandas psicológicas e baixo controle sobre essas, estão em burnout. Ainda, à medida que as demandas psicológicas aumentam, também aumentam o desgaste emocional e a despersonalizaçáo. Por outro lado, quando a demanda psicológica é baixa, o profissional não está em burnout. Outrossim, profissionais de saúde de unidades de perioperatório exercem suas atividades com pouco controle sobre o trabalho e essa realidade interfere diretamente na interface saúde do trabalhador e segurança do paciente. Ademais, considera-se o viés de temporalidade como sendo uma limitação da presente pesquisa, visto que esse relaciona-se aos estudos transversais, o que impediu de estimar a relação de causa e efeito entre exposição e desfecho.

\section{Colaborações}

Munhoz OL, Arrial TS, Barlem ELD, Dalmolin GL, Andolhe R e Magnago TSBS contribuíram com a concepção do projeto, redaçáo do artigo, revisão crítica relevante do conteúdo intelectual e aprovação final da versão a ser publicada.

\section{Referências}

1. Andolhe R, Barbosa RL, Oliveira EM, Costa AL, Padilha KG. Stress, coping and burnout among intensive care unit nursing staff: associated factors. Rev Esc Enferm USP. 2015;49(Esp):58-64.
2. Azevedo BD, Nery AA, Cardoso JF. Estresse ocupacional e insatisfação com a qualidade de vida no trabalho da enfermagem. Texto Contexto Enferm. 2017;26(1):e3940015.

3. Karasek R, Theörell T. Healthy work: stress, productivity, and the reconstruction of working life. New York: Basic Books; c1990.

4. Alves MG, Chor D, Faerstein E, Lopes CS, Wernwck GL. Short version of the "job stress scale": a Portuguese-language adaptation. Rev Saúde Públ. 2004;38(2):164-71.

5. Maslach C. Comprendiendo el Burnout. Cienc Trab. 2009;11(32):37-43.

6. Lautert L. 0 desgaste profissional do enfermeiro [tese]. Salamanca (ES): Faculdade de Psicologia, Universidade Pontifícia de Salamanca; 1995.

7. Maslach C, Jackson S. Maslach Burnout Inventary, Manual. Palo Alto: University of California; 1986.

8. Inoue KC, Versa GL, Murassaki AC, Melo WA, Matsuda LM. Estresse ocupacional em enfermeiros intensivistas que prestam cuidados diretos ao paciente crítico. Rev Bras Enferm. 2013;66(5):722-9.

9. Rodrigues CC, Salvador PT, Assis YM, Gomes AT, Bezerril MS, Santos VE. Estresse entre os membros da equipe de enfermagem. Rev Enferm UFPE. 2017;11(2):601-8.

10. Brum AP. Avaliação do estresse na equipe de enfermagem do turno diurno de um hospital universitário [dissertação]. Porto Alegre: Universidade Federal do Rio Grande do Sul; 2014.

11. Ribeiro RP, Marziale MH, Martins JT, Galdino MJ, Ribeiro PH. Estresse ocupacional entre trabalhadores de saúde de um hospital universitário. Rev Gaúcha Enferm. 2018;39(0):e65127.

12. Magnago TS, Lisboa MT, Griep RH, Zeitoune RC, Tavares JP. Working conditions of nurses: evaluation based on the demand-control model. Acta Paul Enferm. 2010;23(6):811-7.

13. Zanatta $A B$, Lucca SR. Prevalência da síndrome de Burnout em profissionais da saúde de um hospital oncohematológico infantile. Rev Esc Enferm USP. 2015;49(2):253-60.

14. Silveira AL, Colleta TC, Ono HR, Woitas LR, Soares SH, Andrade VL, et al. Síndrome de Burnout: consequências e implicações de uma realidade cada vez mais prevalente na vida dos profissionais de saúde. Rev Bras Med Trab. 2016;14(3):275-84.

15. Neves VF, Oliveira AF, Alves PC. Síndrome de burnout: impacto da satisfação no trabalho e da percepção de suporte organizacional. Psico Porto Alegre PUCRS. 2014;45(1):45-54.

16. Campos IC,AngélicoAP, Oliveira MS, Oliveira DC. Fatores sociodemográficos e ocupacionais associados à síndrome de burnout em profissionais de enfermagem. Rev. Psicol Reflex Crit. 2015;28(4):764-71.

17. Machado RM, Oliveira SP, Ferreira TC, Campos CG, Botti NC, Consolação R. Síndrome de burnout em centro de terapia intensiva infantil da região centrooeste de Minas Gerais. Rev Enferm Centro Oeste Min. 2011;1(2):201-9.

18. Rodrigues CC, Santos VE, Sousa P. Patient safety and nursing: interface with stress and Burnout Syndrome. Rev Bras Enferm. 2017;70(5):1083-8.

19. Guirardello EB. Impacto do ambiente de cuidados críticos no burnout, percepção da qualidade do cuidado e atitude de segurança da equipe de enfermagem. Rev Lat Am Enfermagem. 2017;25:e2884.

20. Fabichak C, Silva-Junior JS, Morrone LC. Síndrome de burnout em médicos residentes e preditores organizacionais do trabalho. Rev Bras Med Trab. 2014;12(2):79-84.

21. Salvagioni DA, Melanda FN, Mesas AE, González AD, Gabani FL, Andrade SM. Physical, psychological and occupational consequences of job burnout: A systematic review of prospective studies. PLoS One. 2017;12(10):e0185781. 